\title{
Sistemas de Compostaje para el Tratamiento de Alperujo
}

\author{
María T. Varnero, Karina Galleguillos y Ronald Rojas \\ Universidad de Chile, Facultad de Ciencias Agronómicas, AGRIMED, Casilla 1004, Santiago- \\ Chile. (e-mail: mvarnero@gmail.com; kgalleguillos@gmail.com; ronaldrojasc@gmail.com)
}

Recibido Feb. 01, 2011; Aceptado Feb. 28, 2011; Versión Final recibida Mar. 29, 2011

\section{Resumen}

Se evaluaron cuatro sistemas de compostaje durante 140 días para el tratamiento aeróbico del alperujo: T1 con pilas $100 \%$ alperujo y volteo manual, T2 con pilas $100 \%$ alperujo y aireación forzada, T3 con pilas $75 \%$ alperujo y $25 \%$ chips de poda y volteo manual, y T4 con pilas $75 \%$ alperujo y $25 \%$ chips de poda con aireación forzada combinada con volteo manual. Los tratamientos con el agente estructurante (T3 y T4) alcanzaron la etapa termofílica al séptimo día; en cambio, T1 y T2 a los veintiún días de compostaje. Sin embargo, esta fase termofílica duró 130 días aproximadamente en T1, T3 y T4: mientras que en T2 duró 30 días, con posterior descenso de las temperaturas en las pilas de compostaje. Esto marca diferencias entre los tratamientos, demostrando que el alperujo no puede ser tratado sólo con aireación forzada, pues este sistema facilita la compactación del residuo.

Palabras clave: compostaje de alperujo, calidad del compost, aireación forzada, volteo manual, agente estructurante

\section{Composting Systems for Olive Residues Treatment}

\begin{abstract}
Four composting systems for aerobic treatment of olive residues were evaluated during 140 days: T1 with windrows $100 \%$ olive residues and manual turning, T2 with windrows $100 \%$ olive residues and forced aeration, T3 with windrows $75 \%$ olive residues and $25 \%$ chips pruning and manual turning, and T4 with windrows $75 \%$ olive residues and $25 \%$ pruning chips with forced aeration combined with manual turning. Treatments with bulking agent (T3 and T4) reached thermophilic phase at the seventh day, whereas T1 and T2 at the twenty-first day of composting. Thermophilic phase lasted approximately 130 days in T1, T3 and T4, while in T2 lasted 30 days. A subsequent drop in the temperature of compost windrows was observed. Noticeable differences among treatments were found demonstrating that olive residues can not be treated exclusively with forced aeration since this system facilitates the compaction of the wastes.
\end{abstract}

Keywords: olive residues composting, compost quality, forced aeration, manual turning, bulking agent 


\section{INTRODUCCIÓN}

Chile ha experimentado un importante aumento en la producción de aceite de oliva extra virgen, estimándose una proyección (CHILEOLIVA, 2009) de 35.000 toneladas para el año 2015. La extracción industrial del aceite de oliva genera una elevada cantidad de residuos y subproductos que requieren una gestión adecuada para minimizar su impacto ambiental (Pozzi et al, 2010). En el caso del sistema de dos fases se genera un residuo orgánico, denominado alperujo (AL), el cual contiene un alto nivel de materia orgánica y nutrientes, pero presenta algunas dificultades en su manejo debido a su elevado contenido de humedad, pequeño tamaño de sus partículas y presencia de sustancias fenólicas hidrosolubles (Albuquerque et al, 2006). Estas últimas características, desaconsejan su utilización directa en suelos como enmienda orgánica, sin un tratamiento previo.

El compostaje es un bioproceso aeróbico que permite la recuperación de los residuos orgánicos heterogéneos, convirtiéndolos en un producto homogéneo de gran calidad Ilamado Compost. La aplicación de este producto a los suelos, permite incrementar la población microbiana existente, aumentando la fertilidad de los mismos (Alburquerque et al, 2007). Sin embargo, si los residuos orgánicos no son tratados adecuadamente pueden presentar efectos inhibitorios en el desarrollo de cultivos, debido a los niveles de fitotoxicidad de las materias orgánicas. Los efectos fitotóxicos se relacionan con diversos factores, entre los cuales se pueden mencionar contenido de metales pesados, contenido de amonio, contenido de sales y ácidos grasos de bajo peso molecular.

Considerando que el AL presenta una alta capacidad de compactación, lo que podría limitar la degradación aeróbica, es necesario agregar algún residuo que actúe como agente estructurante, o bien, aplicar métodos suplementarios de aireación, para mejorar las condiciones aeróbicas de la pila de compostaje.

El objetivo de este estudio fue evaluar distintos sistemas de compostaje para el tratamiento de AL, debido a la alta complejidad estructural que presenta dicho residuo orgánico.

\section{MATERIALES Y MÉTODO}

El estudio de compostaje de $\mathrm{AL}$ se realizó en la Planta Piloto de Compostaje del Centro de Agricultura y Medio Ambiente (AGRIMED), y los análisis químicos y biológicos en el laboratorio de Reciclaje Orgánico de la Facultad de Ciencias Agronómicas de la Universidad de Chile. Las materias primas que se utilizaron fueron alperujo $(A L)$ y como agente estructurante chips de podas (CP), proveniente principalmente de la poda de álamos (Populus $s p$ ), falso pimentero (Schinus molle $\mathrm{L}$ ), entre otros árboles establecidos en la vía publica de la Comuna de la Pintana, Santiago Chile. Para evaluar los sistemas de degradación aeróbica del AL, se realizaron 4 tratamientos (Tabla 1) con 3 repeticiones, donde la unidad experimental fue la pila de Compost.

Tabla 1: Sistemas de tratamiento aeróbico del alperujo.

\section{TRATAMIENTOS}

T1 (Testigo) $\quad 100 \%$ de alperujo con volteo manual periódico.


El tamaño de las pilas de los tratamientos T1 y T3, fue de $1 \mathrm{~m}$ de altura y una base de $1 \times 1 \mathrm{~m}$. Para los tratamientos T2 y T4, se construyó 1 pila con una altura de $1 \mathrm{~m}$ y una base de $3 \times 1 \mathrm{~m}$ $\left(3 \mathrm{~m}^{3}\right)$ en cada caso. Previo a la formación de pilas, se realizó una caracterización química (Tabla 2) y potencial de fitotoxicidad ( $\%$ G) del AL y de CP (TMECC, 2002).

Tabla 2: Caracterización química y potencial de fitotoxicidad de las materias primas.

\begin{tabular}{|c|c|c|c|c|c|c|c|}
\hline $\begin{array}{c}\text { MATERIAS } \\
\text { PRIMAS }\end{array}$ & $\begin{array}{c}\text { Humedad } \\
\%\left(65^{\circ} \mathrm{C}\right)\end{array}$ & $\begin{array}{c}\mathrm{pH} \\
\left(\mathrm{en} \mathrm{H}_{2} \mathrm{O} 1: 5\right)\end{array}$ & $\begin{array}{c}\mathrm{CE} \\
\left(\mathrm{dS} \mathrm{m}^{-1}\right)\end{array}$ & $\begin{array}{c}\mathrm{MO} \\
\left(\mathrm{g} \mathrm{kg}^{-1}\right)\end{array}$ & $\begin{array}{c}\mathrm{N} \text { total } \\
\left(\mathrm{g} \mathrm{kg}^{-1}\right)\end{array}$ & $\mathrm{C} / \mathrm{N}$ & $\begin{array}{c}\% \mathrm{G} \\
\text { rabanito }\end{array}$ \\
\hline Alperujo & 48 & 5,02 & 1,21 & 773 & 7,3 & $59 / 1$ & 37 \\
\hline Chips poda & 12 & 5,76 & 0,68 & 931 & 6,6 & $78 / 1$ & 69 \\
\hline
\end{tabular}

Durante el proceso de compostaje, se registró un control periódico de la temperatura, y del contenido de humedad. En función de los picos de temperaturas se determinó (TMECC, 2002): $\mathrm{pH}$; conductividad eléctrica (CE); materia orgánica (MO); relación carbono - nitrógeno ( $\mathrm{C} / \mathrm{N})$; fitotoxicidad, mediante pruebas de germinación en semillas de rabanito (Raphanus sativus) y estabilidad biológica, mediante respirometría. Los resultados que se exponen de cada tratamiento $(\mathrm{T})$, corresponden a muestras compuestas que se recolectaron el día de inicio (Ti) y el día final (Tf) del proceso de compostaje.

Se realizó un análisis de varianza (ANDEVA) con los datos de materia orgánica, pH y conductividad eléctrica, para determinar si se presentaron diferencias significativas en las características de los productos obtenidos por los diferentes tratamientos. El porcentaje de confiabilidad utilizado fue de un $95 \%$. Posteriormente se realizaron pruebas de comparaciones múltiples para contrastar los tratamientos, cuando existieron diferencias significativas, utilizando un nivel de significancia del $95 \%$.

\section{RESULTADOS Y DISCUSIÓN}

Las mediciones diarias de la temperatura interna de las pilas en proceso de compostaje (Fig. 1), señalaron que la fase termofílica se alcanzó durante los primeros siete días de compostaje en los tratamientos con agente estructurante (T3 y T4). Por su parte los tratamientos T1 y T2 alcanzaron esta fase después de los 21 días de compostaje (Fig. 1). En el tratamiento T3, la temperatura se mantuvo sobre los $45^{\circ} \mathrm{C}$ durante 90 días. En el caso del T4, la fase termofílica se extendió por 125 días aproximadamente, mientras que en el tratamiento T1 ésta fase duró alrededor de 120 días. Estos amplios periodos en rangos termofílicos reflejan que esta fase es muy extensa para este tipo de residuo, debido a los compuestos lipídicos y ácidos orgánicos que presenta. Sin embargo, el tratamiento T2 (alperujo con aireación forzada), tiene aproximadamente una fase termofílica de 30 días lo que evidencia que hay una diferencia con el resto de los tratamientos en el compostaje de alperujo, demostrando que este sistema no es el más adecuado para estos tipos de residuos que tienden a compactarse y formar conglomerados que impiden una adecuada aireación, lo que repercute directamente en el descenso de la temperatura. Por el contrario, en los tratamientos T1 y T3, (con sistema de volteos periódicos), y T4, (con una combinación de aireación forzada y volteos periódicos) existió una mayor oxigenación, lo cual intensificó la acción de los microorganismos termofílicos, favoreciendo las altas temperaturas en las pilas de compostaje, lo que a su vez, permite la degradación de las fracciones orgánicas más resistentes.

No se observaron temperaturas superiores a $70{ }^{\circ} \mathrm{C}$ que pudieran inhibir la actividad microbiana. Las temperaturas máximas alcanzadas en el tratamiento T3, durante los primeros diez días, corresponden a la intensa actividad degradativa por parte de los microorganismos termofílicos sobre los compuestos menos resistentes a la descomposición. 


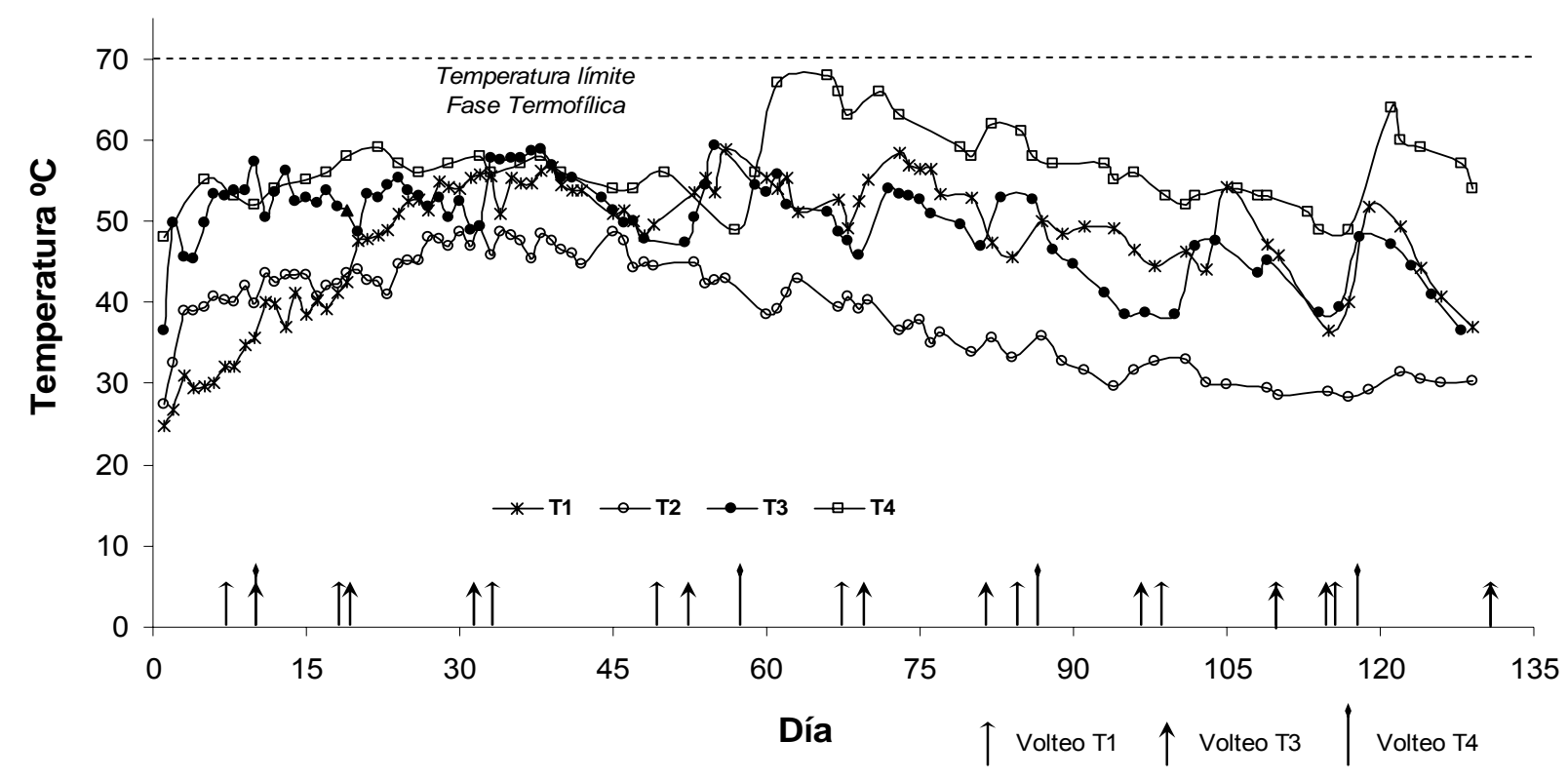

Fig. 1: Evolución de la temperatura interna de la pila de Compost en cada tratamiento durante el proceso de compostaje (Valores promedios). Las flechas indican los días de volteos realizados en cada tratamiento.

En los tratamientos T1, T3 y T4 se observó la aparición de hongos aproximadamente al séptimo día de tratamiento, cuando la temperatura se encontraba entre los 30 y $50^{\circ} \mathrm{C}$. Estos hongos predominaron principalmente a los $30 \mathrm{~cm}$ de profundidad y presentaron un color blanco.

Los picos de temperatura que se observaron en los tratamientos T1, T3 y T4 son producto de los volteos realizados en las pilas, que desencadenan alzas de temperaturas con posteriores descensos de las mismas. Estas alzas y posteriores descensos no se observaron en el tratamiento T2 con aireación forzada.

La NCh 2880 (INN, 2004), señala que se debe pasar por una etapa termofílica de 15 días con un sistema de volteo mecánico o de al menos tres días consecutivos en sistemas con aireación forzada. Posteriormente a esta fase, se evidencia un descenso de la temperatura en las pilas, iniciándose así la etapa mesofílica. La humedad óptima requerida para que predomine una degradación aeróbica en el proceso de compostaje, se sitúa entre $30-45 \%$. Con valores superiores a los indicados se produce una aireación deficiente porque disminuye el aire que circula, favoreciendo los procesos anaeróbicos. Si la humedad es inferior al $10 \%$, desciende la actividad microbiológica y el proceso de compostaje se hace muy lento (Varnero et al, 2004). Para controlar eventuales pérdidas de agua que pudieran inhibir el metabolismo microbiano, debido a las altas temperaturas ambientales que se presentaron durante el desarrollo del estudio, se realizaron riegos frecuentes en las pilas con agua de pozo. No hubo control de lixiviados, pero posiblemente los riegos pudieron producir el arrastre de sales solubles contenidas en los residuos tratados.

El porcentaje de agua presente en la etapa inicial (Tabla 3), fue similar en los tratamientos T1 y T2 con un $45 \%$ de humedad. Este porcentaje de humedad, disminuye en los tratamientos con agente estructurante, T3 y T4, debido al menor contenido de agua que tienen los chips de poda (12\% de humedad), lo que incide en el contenido de la mezcla de materiales que se prepara al momento de construir las pilas de Compost. Los valores promedios iniciales de $\mathrm{pH}$ de todos los tratamientos, están en el rango moderadamente ácido y al término del proceso de compostaje, sólo T4 queda en $\mathrm{pH}$ neutro. En relación a la conductividad eléctrica, se puede señalar que todos los tratamientos presentaron valores iniciales dentro de lo establecido por la NCh 2880-2004, para un Compost clase A, con lo cual, se podría descartar posibles problemas de fitotoxicidad atribuible a salinidad. Cabe mencionar, que el valor de CE inicial de T3 respecto de T4 varió, aún presentando 
la misma mezcla inicial, posiblemente debido a problemas en la homogeneización de los materiales y al origen heterogéneo de las podas utilizadas. Los bajos niveles de salinidad que presentaron los distintos tratamientos, disminuyeron entre un 36 y $55 \%$ respecto del inicial (Tabla 3), situándose en valores cercanos a $1 \mathrm{dS} \mathrm{m}^{-1}$, al finalizar la fase de maduración. Respecto al contenido de materia orgánica inicial, se observó que T3 y T4 presentaron diferencias, aún cuando la composición teórica inicial es la misma. Esto se podría atribuir a problemas de homogeneización de los materiales y al origen heterogéneo de las podas utilizadas, por lo que se recomienda utilizar un método que permita mejorar las condiciones de mezcla. Durante el proceso de compostaje, se observó una disminución en el contenido de materia orgánica, debido a las reacciones de descomposición aeróbica que generan principalmente dióxido de carbono y agua. Se observa una disminución en el valor de la materia orgánica de 20, 13 y $16 \%$ en los tratamientos T1, T3 y T4 respectivamente, siendo más pronunciada en los primeros quince días del proceso de compostaje. En cambio, en el tratamiento T2 no se observa una disminución clara de la materia orgánica, no superando el $2 \%$, lo que podría deberse a los problemas de compactación que impiden un adecuado proceso de degradación.

Tabla 3: Caracterización química de las materias procesadas mediante compostaje, al inicio y término del ensayo.

\begin{tabular}{|l|c|c|c|c|c|c|c|c|}
\hline PARÁMETROS & T1i & T2i & T3i & T4i & T1f & T2f & T3f & T4f \\
\hline Humedad \% $\left(65^{\circ} \mathrm{C}\right)$ & 45 & 45 & 35 & 40 & 41 & 45 & 42 & 40 \\
\hline pH & 5,45 & 5,44 & 5,09 & 5,40 & 6,54 & 5,21 & 6,06 & 7,10 \\
\hline C.E. $\left(\mathrm{dS} \mathrm{m}^{-1}\right)$ & 1,16 & 1,23 & 1,17 & 2,90 & 0,70 & 0,70 & 0,70 & 1,34 \\
\hline M.O. $\left(\mathrm{g} \mathrm{kg}^{-1}\right)$ & 751 & 734 & 762 & 864 & 598 & 719 & 670 & 730 \\
\hline
\end{tabular}

Con relación al análisis estadístico se observa que en cuanto a los valores de $\mathrm{pH}$, los tratamientos no presentan diferencias significativas para el producto final. Respecto a la conductividad eléctrica y niveles de materia orgánica, el producto final de los distintos tratamientos presenta diferencias significativas, por lo tanto, al aplicar la prueba de comparaciones múltiples el producto obtenido en T4 es significativamente distinto a los otros tres productos, para estos análisis.

La calidad química y biológica de un residuo orgánico valorada a través del índice de madurez, considera tres características esenciales (Varnero et al, 2004): a) La relación C/N, para discriminar materiales con mayor probabilidad de inmovilizar N. b) La Estabilidad Biológica, medida por respirometría, la cual excluye materiales que se encuentran en activa descomposición microbiana porque contienen un nivel de carbono que sustenta la actividad microbiana. c) El Potencial de Fitotoxicidad, evaluado mediante bioensayos para establecer la presencia de metabolitos fitotóxicos, como consecuencia de una biodegradación que se encuentra aún en fases intermedias.

Estos compuestos pueden tener efectos inhibitorios tanto en la germinación de las semillas, como en el crecimiento de ciertas plantas. A través del bio-ensayo, se logra detectar la presencia de sustancias tóxicas en diversos tipos de Compost y en materiales en proceso de compostaje, para lo cual se utilizan especies indicadoras sensibles (Varnero et al, 2006), de rápido crecimiento y fácil manejo. Estas especies permiten advertir la presencia de sustancias fitotóxicas mediante los efectos negativos que provocan sobre la germinación y crecimiento de las semillas. Las especies más apropiadas para este efecto (Varnero et al, 2007) son rabanito (Raphanus sativus), lechuga (Lactuca sativa) y berro de huerto (Lepidium sativum). La Normativa Chilena de Compost - NCh 2880 (INN, 2004) establece al rabanito como la especie adecuada para realizar estos bio-ensayos, calificando a un Compost maduro si éste muestra un porcentaje de germinación igual o superior al $80 \%$. 
La Normativa Chilena de Compost - NCh 2880 (INN, 2004) establece que los materiales orgánicos compostados se pueden denominar "Compost", cuando cumplen con los parámetros que definen el Índice de Madurez. Al respecto, se puede mencionar que el tratamiento T2 (Tabla 4) no logró alcanzar una relación $\mathrm{C} / \mathrm{N}$ óptima durante el proceso, mientras que los tratamientos $\mathrm{T} 1$, T3 y T4 presentaron una relación $\mathrm{C} / \mathrm{N}$ igual o inferior a 30 (Tabla 4), a contar de los 90 días de compostaje aproximadamente. Esto coincide con el inicio de la etapa de enfriamiento y maduración del proceso de compostaje, lo que se explicaría porque parte del carbono es liberado constantemente como $\mathrm{CO}_{2}$, mientras que el nitrógeno es reciclado, lo que refleja la descomposición de la materia orgánica y su estabilización.

Tabla 4: Valores promedios obtenidos en las materias bioprocesadas al término del proceso de compostaje, para los parámetros de Índice de Madurez: relación $\mathrm{C} / \mathrm{N}$, respirometría y porcentaje de germinación en semillas de rabanito (Raphanus sativum).

\begin{tabular}{|c|c|c|c|c|c|}
\hline PARÁMETROS & T1 & T2 & T3 & T4 & $\begin{array}{l}\text { Rango de aceptación } \\
\text { (NCh 2880-2004) }\end{array}$ \\
\hline Relación C/N & $30 / 1$ & $48 / 1$ & $30 / 1$ & $21 / 1$ & $\begin{array}{l}\text { Clase } A \leq 25 \\
\text { Clase } B \leq 30\end{array}$ \\
\hline $\begin{array}{l}\text { Respirometría } \\
\left(\mathrm{mg} \mathrm{C}-\mathrm{CO}_{2} / \mathrm{g} \mathrm{MO} / \text { día }\right)\end{array}$ & 3,5 & 8,5 & 4,4 & 4,2 & $\begin{array}{l}\leq \text { a } 8 \mathrm{mg} \text { de } \mathrm{C}-\mathrm{CO}_{2} \mathrm{~g}^{-1} \\
\mathrm{MO} \mathrm{día}^{-1}\end{array}$ \\
\hline $\begin{array}{l}\text { Germinación en semillas de } \\
\text { rabanito (\%) }\end{array}$ & 92 & 45 & 94 & 92 & $\geq$ a $80 \%$ \\
\hline
\end{tabular}

Al comparar los valores de estabilidad biológica obtenidos para cada tratamiento (Tabla 4) con los valores de referencia de la $\mathrm{NCH} 2880$ (INN, 2004), que señala que deben ser $\leq 8 \mathrm{mg} \mathrm{C}-\mathrm{CO}_{2} / \mathrm{g}$ $\mathrm{MO} /$ día, se puede apreciar que sólo el tratamiento T2, queda fuera del rango exigido por esta Norma. Esto se podría atribuir a las temperaturas observadas durante el proceso de compostaje, las que no superaron los $55^{\circ} \mathrm{C}$, con lo cual existirían fracciones orgánicas sin descomponer. Por otra parte, los porcentajes de germinación (Tabla 4) muestran que aproximadamente a los 84 días de tratamientos, sólo el material obtenido de T2; revela la existencia de metabolitos fitotóxicos severos, porque presenta una germinación igual a 45\%. En cambio, en los tratamientos T1, T3 y T4, se supera el $80 \%$ exigido como mínimo.

\section{CONCLUSIONES}

De acuerdo al estudio realizado, el tratamiento del alperujo con aireación forzada (T2) resultó inadecuado probablemente por que facilitó la compactación del alperujo, lo que debió incidir negativamente en el desarrollo de la fase termofílica del proceso de compostaje, afectando la calidad del producto final obtenido. Esto se reflejó en los valores de la relación $\mathrm{C} / \mathrm{N}$, estabilidad biológica y potencial de fitotoxicidad de los composts obtenidos.

La incorporación de aireación forzada combinada con volteo, a pilas de compostaje de alperujo con un agente estructurante, permitió realizar una menor cantidad de volteos y cumplir con los parámetros exigidos por la NCh 2880-2004 en cuanto al Índice de Madurez de materiales procesados, obteniéndose un Compost clase A.

\section{REFERENCIAS}

Alburquerque, J.A. y otros tres autores, Effects of bulking agent on the composting of "alperujo", the solid by-product of the two-phase centrifugation method for olive oil extraction, Process Biochemistry: 41, 127-132 (2006). 
Alburquerque, J.A. y otros tres autores, Effects of a compost made from a solid by-product ("alperujo") of a two-phase centrifugation system for olive oil extraction and cotton gin waste on growth and nutrients content ryegrass (Lolium perenne L.), Bioresource Technology: 98, 940 - 945 (2007).

CHILEOLIVA, Informe anual del mercado nacional chileno del olivo (en línea), 2009. Disponible: http// www.chileoliva.com. Acceso: 30 de Noviembre (2010).

NCh 2880-2004, Norma Chilena de Compost 2880-2004: Compost - Clasificación y requisitos, Instituto Nacional de Normalización (INN), 23 p, Santiago, Chile (2004).

Pozzi, M. y otros tres autores, Posibilidad de usar el alpechín en fertilización de tierras agrícolas, Información Tecnológica: 21(4), 117-123 (2010).

TMECC, Leege P.B. and W.H. Thompson (Eds.), Test Methods for Examination of Composting and Compost The U.S Composting Council Research and education Foundation. Bethesda, Maryland, US. (2002).

Varnero, M.T. y otros tres autores, Evaluación de lodo fresco y compostado como materia prima para la elaboración de sustrato, Actas del Simposio de las Ciencias del Suelo "Residuos Orgánicos y su Uso en Sistemas Agroforestales", 361- 365, Temuco - Chile, 5 a 6 de Agosto (2004).

Varnero, M.T. y otros tres autores, Evaluación de especies sensibles a metabolitos mediante bioensayos de germinación, El Medioambiente en Iberoamérica: Visión desde la Física y la Química en los albores del Siglo XXI, Editor Juan F. Gallardo Lancho, Sociedad Iberoamericana de Física y Química Ambiental, Badajoz, España. Tomo III, 363 -369. (2006).

Varnero, M.T., C. Rojas y R. Orellana, Índices de fitotoxicidad de residuos orgánicos durante el compostaje, Revista de la Ciencia del Suelo y Nutrición Vegetal, 7 (1), 28 - 37 (2007). 
